\title{
Outcome of Non-Traumatic Surgical Acute Abdomen in Nekemte Referral Hospital Southwest Ethiopia: A Retrospective Cross-Sectional Study
}

\section{Zinabu Ayenew ${ }^{1}$, Abraham Tamirat Gizaw ${ }^{2 *}$, Desta Workneh ${ }^{3}$ and Netsanet Fentahun}

${ }^{1}$ Department of Obstetrics and Gynecology and Surgery, Jimma University, Jimma, Ethiopia

${ }^{2}$ Departments of Health Education and Behavioral Sciences, Jimma University, Jimma, Ethiopia

${ }^{3}$ Departments of Nursing and Midwifery, Jimma University, Jimma, Ethiopia

\section{Abstract}

Background: Acute abdomen is an acute onset of abdominal disease entities that require immediate surgical intervention in most of the cases. The causes of non-traumatic surgical acute and their relative incidence varies in different populations.

Objective: To determine the magnitude and the common causes as well presenting features and the outcome of non-traumatic surgical acute abdomen (In Nekemte Referral Hospital, Oromia region, Ethiopia)

Methods: A retrospective cross sectional study was conducted using data available at Nekemte Referral Hospital from January 2011 to December 2013. We examined 295 records of the patients from the medical records. Data were collected using checklist based on registration books. The data were entered and analyzed using SPSS version 20.0 statistical package. The association between the independent and dependent variable was assessed by bivariate and multivariate regression analyses. A $95 \% \mathrm{Cl}$ and $p$-value of $\leq 0.05$ were used to determine independent predictors of outcome of non-traumatic surgical acute abdomen.

Results: Out of 295 patients, 230 were males and 65 were females. The age ranged from 6 months to 80 years with a mean age of $33.7 \pm 18.6$ years. Out of $295,57.6 \%$ was rural and $46.4 \%$ were urban dwellers. The most common cause of acute abdomen was acute appendicitis 140(47.4\%) followed by bowl obstruction 118(40.0\%). Age, Residence, Duration of illness and length of hospital stays are independent predictors of management outcomes of non-Traumatic acute abdomen.

Conclusion: Complications were more in cases from rural area due to delay at presentation and no operation facility and surgeons for solutions.

Keywords: Non-traumatic; Surgical; Outcome; NRH

\section{Introduction}

Acute abdomen is the most common presenting surgical emergency. It has been estimated that at least $50 \%$ of general surgical admissions are emergencies and $50 \%$ of them present with acute abdominal pain. Acute abdomen' is a term used to encompass a spectrum of surgical, medical and gynecological conditions, ranging from the trivial to the life-threatening, which require hospital admission, investigation and treatment. The acute abdomen may be defined generally as an intraabdominal process causing severe pain requiring admission to hospital, and which has not been previously investigated or treated and may need surgical intervention [1].

Intestinal obstruction has been the leading cause of acute abdomen in several African countries where as acute appendicitis is the most frequently seen cause in the developed world $[2,3]$. The leading causes of intestinal obstruction in Africans have mostly been hernia and volvulus whereas adhesions are most frequent in the developed world $[3,4]$. There are, however, some African studies which are pointing to a change in these established patterns $[5,6]$.

The mortality rate following acute abdominal surgical emergencies is still high. The overall mortality was $4.2 \%$ and the rate increased significantly in patients aged $>60$ years. Postoperative mortality was $5.8 \%$ while mortality rate in non-operated patients was $2.8 \%$. The causes of preoperative death included perforated peptic ulcer, abdominal malignancies (15.4\%) and urgent colonic resections (9.4\%). Emergency procedures generally are associated with increased morbidity and mortality rate in elderly patients (the overall mortality was $22 \%$ ) $[7,8]$.

Study done in Sinai Hospital, in Tehran, Iran shows that 139 patients diagnosed with acute abdomen underwent emergency Laparotomy.
According to this research, result acute appendicitis was the most common cause of acute abdomen (56.8\%). Acute appendicitis was the etiology of acute abdomen in $67 \%$ of male and $38.8 \%$ of female patients. Other common causes of acute abdomen were $14.4 \%$ peritonitis among which 5.7\% resulted from PPUD, (3.5\%) were perforated appendicitis, $1.4 \%$ pancreatitis, $7.2 \%$ Cholecystitis. Bowel obstruction $7.2 \%$ of which $2.2 \%$ adhesion and volvulus each, $1.4 \%$ incarcerated hernia and only $0.7 \%$ case of imagination found [9].

Study conducted on 190 cases at Kamuzu Central Hospital (KCH) in Lilongwe, Malawi, during the calendar year 2008 Sixty-nine percent were male. The average age was 35 (median 32, range10-84). The youngest subject was 10 , and 10 subjects were under the age of 18 . The most common etiologies were appendicitis $(22 \%)$, intestinal volvulus (17\%), perforated peptic ulcer (11\%) and small bowel perforation (11\%). The overall mortality rate associated with peritonitis was $15 \%$, with the highest mortality rates observed in solid organ rupture (35\%), perforated peptic ulcer (33\%), primary/idiopathic peritonitis $(27 \%)$,

*Corresponding author: Abraham Tamirat Gizaw, Jimma University, College of Public Health, Department of Health Education and Behavioral Science P.O.Box-378, Jimma, Ethiopia, Tel: +251 47111 1458; E-mail: abrishntamirat@ gmail.com

Received November 15, 2016; Accepted December 07, 2016; Published December 16, 2016

Citation: Ayenew Z, Gizaw AT, Workneh D, Fentahun N (2016) Outcome of Non-Traumatic Surgical Acute Abdomen in Nekemte Referral Hospital Southwest Ethiopia: A Retrospective Cross-Sectional Study. Surgery Curr Res 7: 282. doi: 10.4172/2161-1076.1000282

Copyright: @ 2016 Ayenew Z, et al. This is an open-access article distributed under the terms of the Creative Commons Attribution License, which permits unrestricted use, distribution, and reproduction in any medium, provided the original author and source are credited. 
tubo-ovarian abscess (20\%) and small bowel perforation (15\%). Factors associated with increased mortality include age, gender, symptoms (constipation, vomiting) and symptom duration [10].

Typhoid ileal perforation still remains a very severe condition in tropical countries. Its incidence ranges from $0.9 \%$ to $39 \%$, with a mortality rate, which remains very high. Primarily, the mortality and the morbidity rate do not depend on the surgical technique, but rather on the general status of the patient, the virulence of the salmonella and the duration of disease evolution before surgical treatment. That is why, it is so important to provide adequate pre-operative management associating aggressive resuscitation with antibiotic therapy. In the literature, it is usually advocated that the last $60 \mathrm{~cm}$ of the ileum present a high concentration of Peyer's patches whose infection is a source of intestinal perforation $[3,11]$.

Four year retrospective study at Gondar University Hospital shows that emergency laparotomies for non-traumatic acute abdomen were $43.3 \%$ of all laparotomies. The leading operative diagnoses were SBO (43.4\%), appendicitis (34.6\%) and LBO (11.5\%). Majority of surgically treated acute abdomen patients were from rural areas $(58.2 \%)$. Abdominal pain (100\%), Vomiting (90.3\%), abdominal distension (58.3\%) and constipation (55.3\%) were the commonest symptoms in patients with acute abdomen. Seventy-seven (26.6\%) of operated patients had early (in-hospital) postoperative complications [4].

\section{Methods}

\section{Study area and design}

This was a retrospective cross sectional study using data available at Nekemet Referral Hospital during the 3-year period (January 2011 to December 2013). The hospital is located in Horogudru West Shewa Zone, Ethiopia about 331 Kilometers from Addis Ababa. Nekemet Referral Hospital is a public health facility and run by the government. Currently NRH 178 beds used for the inpatient services.

\section{Study sample}

The study sample included all 295 medical records of on pattern of emergency operations performed for non-traumatic surgical acute abdomen 2011-2013. However, incomplete records were excluded.

\section{Study variables}

The outcome variables for this study were Postoperative complications and Outcome on discharge. Age, Sex, Place of residence, occupation, Operative diagnosis, Type of procedure, Duration of illness before operation, Clinical features, occupation, duration of hospital stay were extracted as variable from the record.

\section{Data extraction}

The records include information on subjects' demographics, vital signs, laboratory test result, prescribed drugs list, hospital stay etc. To extract data from records, we developed a checklist containing three parts, namely socio demographic variables and cause of Emergency surgical and surgical outcomes. To ensure the quality and consistency, we trained researchers on the meanings of each item on the checklist and how to extract data. Then four trained health professionals extracted data from the records related to each item in the checklist.

\section{Data processing, analysis, interpretation and presentation}

After data collected, it was coded, entered and cleaned using computer software SPSS version 20. Descriptive statistics were presented by frequency tables and figures. On bivariate analysis $\mathrm{p}$-value $<0.25$ was used as a candidate for multivariate logistic regression. A 95\% CI and p-value of $\leq 0.05$ were used to determine independent predictors of outcome of non-traumatic surgical acute abdomen.

\section{Ethical considerations}

Ethical clearance was obtained from the ethical review committee of Jimma University. A formal letter was written from the coordinator of Integrated Emergency Obstetrics/Gynecology and surgery to the hospital administrator office. The Hospital medical director permitted us to conduct the study. The data were collected by review of the registration books using structured checklists.

\section{Results}

\section{Socio-demographic characteristics}

Two hundred ninety five cases were retrieved which made the basis of this study. There were 230(78\%) male participants. Age ranged from 6 months to 80 years with a mean age of $33.7 \pm 18.65$ years. Out of $295,158(57.6 \%)$ patients were rural dwellers. More than $51.5 \%$ of patients came within 2 days of illness and less than $8.1 \%$ came more than 7 days of illness (Table 1).

\section{Causes and symptom of acute abdomen}

The most common cause of acute abdomen was acute appendicitis $140(47.4 \%)$ followed by bowl obstruction $118(40.0 \%)$. The commonest presenting compliant was abdominal pain $(90.1 \%)$ followed by vomiting (7.8\%) (Table 2).

\section{Appendicitis}

Acute appendicitis accounts the majority of the cases 74(52.8\%) of appendicitis followed by Appendiceal abscess 38 (27.1\%) and perforated appendicitis $28(20 \%)$. Appendiceal abscess was high in elder age groups $(>60$ years $=50 \%$ ) where as low in 1 st decade and Perforated appendicitis was high in two extreme age groups ( $42.86 \%$ in 1 st decade and $33.35 \%$ in $>6$ th decades).

Wound infection was the commonest post-operative complication of appendicitis $14(10 \%)$ followed by pneumonia $3(2.1 \%)$ and sepsis 1 $(0.7 \%)$ from total appendicitis cases there were $3(2.2 \%)$ deaths of these $66.6 \%$ were from age group of $>60$ years and those who came after 7 days.

\section{Obstructions}

Small bowel obstruction: Small bowel obstruction was the leading cause of bowel obstruction which accounts 75(63.5\%) of bowel obstruction of which $43(57.3 \%)$ were primary small bowel volvulus, 13(17.3\%) were hernia, 12(16\%) were adhesion/band and 6(8\%) were intussusceptions. The common symptoms were abdominal pain and vomiting in $100 \%$, constipation and distension each seen in (94.67\%) and $(91.99 \%)$ of patients respectively.

Out of 75 small bowel volvulus, $88 \%$ were males and $12 \%$ were females, and majority of cases were in the 1st decade especially intussusceptions was completely in this age group. Fifty four (72\%) of Small Bowl Obstruction cases were found to be viable and 21(28\%) were nonviable. Of 21 cases of nonviable Small Bowl Obstruction, 14 (66.7\%) and 7(33.3\%) were managed by REEA and RESA respectively and the viable ones managed accordingly.

Among 43 cases of primary small bowel volvulus, 32(74.4\%) cases were viable and $11(25.58 \%)$ were nonviable for which derotation plus 
Citation: Ayenew Z, Gizaw AT, Workneh D, Fentahun N (2016) Outcome of Non-Traumatic Surgical Acute Abdomen in Nekemte Referral Hospital Southwest Ethiopia: A Retrospective Cross-Sectional Study. Surgery Curr Res 7: 282. doi: 10.4172/2161-1076.1000282

Page 3 of 5

\begin{tabular}{|c|c|c|}
\hline Variable & Frequency & Percent \\
\hline \multicolumn{3}{|c|}{ Age } \\
\hline$<14$ years & 65 & 22 \\
\hline $15-24$ years & 35 & 11.9 \\
\hline $25-34$ years & 35 & 11.9 \\
\hline $35-44$ years & 41 & 13.9 \\
\hline $45-55$ years & 30 & 10.2 \\
\hline$>55$ years & 89 & $30 . .2$ \\
\hline \multicolumn{3}{|c|}{ Sex } \\
\hline Male & 230 & 78 \\
\hline Female & 65 & 22 \\
\hline \multicolumn{3}{|c|}{ Residence } \\
\hline Rural & 158 & 53.5 \\
\hline Urban & 137 & 46.5 \\
\hline \multicolumn{3}{|l|}{ Occupation } \\
\hline Farmer & 142 & 48.1 \\
\hline Housewife & 16 & 5.4 \\
\hline Merchant & 21 & 7.1 \\
\hline Student & 84 & 28.5 \\
\hline Governmental employer & 32 & 10.8 \\
\hline Total & 295 & 100 \\
\hline
\end{tabular}

Table 1: Socio-demographic characteristics of patients with acute abdomen in Nekemte Referral Hospital from January 2011 -December 2013.

\begin{tabular}{|c|c|c|}
\hline $\begin{array}{c}\text { Cause and Symptom Acute } \\
\text { Abdomen }\end{array}$ & Percent \\
\hline \multicolumn{2}{|c|}{ Nouse of cases } \\
\hline \begin{tabular}{c|c|} 
Appendicitis \\
Bowel obstruction
\end{tabular} & 470 \\
\hline $\begin{array}{c}\text { Peritonitis other than gangrenous } \\
\text { obstruction and perforated } \\
\text { appendicitis }\end{array}$ & 118 & 12.2 \\
\hline Others & 36 & 0.3 \\
\hline & 1 & 90.1 \\
\hline Abdominal Pain & Symptoms & 7.8 \\
\hline Vomiting & 266 & 2.1 \\
\hline Distention & 6 & \\
\hline
\end{tabular}

Table 2: Frequency of the causes of emergency surgically treated non traumatic surgical acute abdomen in NRH during the year of January2011 -December 2013 $(n=295)$.

milking and REEA done respectively. Thirty eight cases were males and 5 were females with 2:1 similar ratio of male to female and rural to urban. The 2nd leading cause of SBO was hernia and 9(69.2\%) were viable $4(39.7 \%)$ were strangulated for which reduction plus defect repair and REEA done respectively. All of these are males and from rural.

Eight cases of adhesion/band were viable and 4 were nonviable. The sex distribution was almost equal and rural-urban ratio was 3:1. All of viable cases managed by simple adhesionolysis and band release whereas nonviable ones by REEA. All of 6 cases of intussusceptions were ilieo-colic and 5 were males and from rural and 1 female and from urban and all of were in 1st decade. Five of these were viable and 1 nonviable managed by simple reduction and RESA respectively. Wound infection (4), leak (4) and sepsis (2) were the main early post-operative complications in SBO. There were $2(2.7 \%)$ deaths of which $1(4.7 \%)$ was from nonviable SBO and $1(1.8 \%)$ from viable $\mathrm{SBO}$.

Large bowel obstruction: Sigmoid volvulus was the leading case of LBO 37(86.0\%) followed by ilieo-sigmoid knotting 5(11.6\%) and colorectal CA $1(2.3 \%)$. The main presenting symptoms were abdominal pain (98\%), constipation (100\%), distension (100\%) and vomiting (95.35\%) of patients. Among 43 cases of LBO, 40(93.0\%) were males and $3(6.9 \%)$ were females with rural-urban ratio of $3: 1$ and $28(65.1 \%)$ were nonviable and $15(34.8 \%)$ were viable.

Twenty-five (89.2\%) of nonviable LBO and were managed by Hartman's colostomy. Colostomy and ilieo-colic anastomosis was done for other 3(10.7\%) cases. Most of viable LBO (11 cases) was managed by simple derotation and deflation while for 4 cases primary REEA done. Wound infection, sepsis, pneumonia accounts 2 in each equally. There were 2 deaths (4.65\%) all of were after Hartman's colostomy done and from rural (Table 3 ).

Peritonitis: There were 107 cases that developed peritonitis at the time of operation from different sources. Forty eight (44.8\%) of cases were from gangrenous bowel obstructions, 22(20.5\%) perforated appendicitis, 14(13.0\%) perforated PUD and 11(10.2\%) from typhoid perforation while $11(10.2 \%)$ were primary peritonitis. Majority of cases of peritonitis were those who came late before operation.

The main presenting features of peritonitis were abdominal pain (100\%), vomiting (97.65) constipation (78\%), and nausea $(69.87 \%)$. Among perforated PUD, 11 were from rural and 3 urban and all were males. Majority of cases were in the age group of 40-60 years. All managed by omental patch and there was one death by the complication of sepsis.

Most cases of typhoid perforation were from rural and in the age group of 1 st and 2 nd decades. Male to female ratio was 1:2 and repair done for eight $(72.7 \%)$ cases where as REEA done for three $(27.2 \%)$. There was 1death from repair due to sepsis secondary to leak. Ten of 11 cases of primary peritonitis were from rural and the male to female ratio was $1: 2$. Six (54.5\%) managed by lavage plus appendectomy and for five (45.4\%) lavage only done. The total deaths among patients who had peritonitis at time of operation were six (5.6\%).

Predictors of management outcomes of non-traumatic acute abdomen: Numerous associations were found to be significant in the bivariate analysis. Therefore, a multivariable approach was applied to determine which factors best explained and predict management outcome of patient. Patient who were $<14$ years of age were 15.4 times more likely to have good management outcome as compared with other age group [AOR=15.4, 95\% CI (1.61-19.98)]. Patient who came from rural area 5 times higher to have bad management outcome as compared to patient who came from urban [(AOR=7.5, 95\% CI (1.4638.46)]. Patient who had $>2$ days duration of illness has 3.8 times higher

\begin{tabular}{|c|c|c|c|}
\hline Cause & No. of cases & $\begin{array}{c}\text { Percent from } \\
\text { SBO }\end{array}$ & $\begin{array}{c}\text { Percent from } \\
\text { total obstruction }\end{array}$ \\
\hline Small Bowl Instruction (SBO) \\
\hline Small bowel volvulus & 43 & 57.3 & 36.4 \\
\hline Hernia & 13 & 17.3 & 11 \\
\hline Adhesion/band & 12 & 16 & 10.1 \\
\hline Intussusceptions & 6 & 8 & 5.1 \\
\hline Other & 1 & 1.3 & 0.9 \\
\hline Total & 75 & 100 & 63.6 \\
\hline & Large Bowl Obstruction (LBO) & \\
\hline Sigmoid volvulus & 37 & 86 & 31.3 \\
\hline Ilieo-sigmoid knotting & 5 & 11.6 & 4.2 \\
\hline Colorectal Ca & 1 & 2.3 & 0.8 \\
\hline Total & 43 & 100 & 36.4 \\
\hline
\end{tabular}

Table 3: Frequency of causes of small bowl obstruction and large bowl obstruction in NRH from January2011 -December 2013. 
Citation: Ayenew Z, Gizaw AT, Workneh D, Fentahun N (2016) Outcome of Non-Traumatic Surgical Acute Abdomen in Nekemte Referral Hospital Southwest Ethiopia: A Retrospective Cross-Sectional Study. Surgery Curr Res 7: 282. doi: 10.4172/2161-1076.1000282

Page 4 of 5

more likely unfavorable outcome as compared to patient who came with $<2$ days of duration $[(\mathrm{AOR}=4.33,95 \% \mathrm{CI}(1.03-18.12)]$.

Length of hospital stay has also significant statistical association with management outcome [(AOR=0.21, 95\%CI (0.06-0.73)]. Patients who stayed for less than 5 days are less likely to develop unfavorable outcome as compared with patients stayed for greater than 5 days (Table 4).

\section{Discussion}

There are very few studies done on the general pattern of acute abdomen in Ethiopia and few studies on specific causes of acute abdomen. In this study emergency surgical operation performed for 295 non traumatic surgical acute abdomens and of these, 230(77.97\%) were males and 65(22.03\%) were females. Seventy percent of all were rural and 30\% were urban residences. Majority of patients were in their 2nd and 3rd decades of life, which was similar with previous studies done in TAH and other African countries. This may be due to the flatus theory that males pass their time in out home duties that restricts them to pass flatus and feces for long time and loaded feeding habit per cycle of meal of males. But the opposite is true for females $[7,12,13]$.

Almost similarly with study in Gondar University Hospital, abdominal pain $(99.6 \%)$, vomiting $(95.2 \%)$, constipation $(59.3 \%)$ and abdominal distension (56.8\%) were the commonest symptoms in patients with acute abdomen [8]. Acute appendicitis was found to be the leading cause of non-traumatic surgical acute abdomen leading to emergency operation in this study. The majority of the cases with acute appendicitis were from urban and in their 2nd and 3rd decades of life with male to female ratio 3:1 which agree with other studies done in TAH, ZMH, and Sina Hospital $[7,9,12]$ but contrast to study done in Gondar University and Yirgalem Hospitals which showed that intestinal obstruction was the leading cause of acute abdomen. This may be explained by due to diet and socio-economic factors that may or may not differ in different areas $[4,8]$.

Bowel obstruction was the second most common cause of nontraumatic acute abdomen with $40 \%$ of which $75(63.5 \%)$ SBO followed by LBO 43 (36.4\%) which was similar with study done in TAH. Primary small bowel volvulus accounting $43(57.3 \%)$ from SBO was the leading cause. This contradicted with studies done in TAH, Gondar University Hospital which has shown that adhesion was the leading cause of small bowel obstruction. It is explained by that since adhesion is a secondary problem, (occurs in patients who has history of previous surgery), there may be high operation rate in catchments of TAH and Gondar university. In this study hernia and adhesion were found to be 2 nd and 3 rd causes of SBO. Out of 75 cases of SBO, 21(28\%) were nonviable and managed by resection and anastomosis $[4,7]$.

This was most seen in patients who presented late, more than 3 days of duration of illness. This is because when the duration of time increases blood perfusion of bowel decreases finally to death of bowel. Similar with TAH, in this study sigmoid volvulus was found to be the leading cause of colonic obstruction (86.0\%). In this study most of patients developed peritonitis at time of operation and most of them resulted from gangrenous bowel obstruction (42.5\%) followed by perforated appendicitis (20.3\%), perforated PUD (12.9\%), typhoid perforation(10.1\%) and others (10.1\%) were primary peritonitis. However contrarily, study on Sina Hospital, showed that $14.4 \%$ of patients developed peritonitis, among which 5.7\% resulted from PPUD, $3.5 \%$ of from perforated appendix. This may be due to late presentation of patients because of different reasons like lack of health awareness, inaccessibility of health institutions where operation is not performed and no trained health staffs who did operations in rural areas [14].

Similar to study in TAH, Peritonitis was highly seen in patient who came late ( $>52.3 \%$ in 2 days) and from rural areas $(83.18 \%)$ and the reason for late presentation to the institution delivering the surgical treatment needs to be studied further [7].

More than $49(16.1 \%)$ surgically treated non traumatic acute abdomen patients had one or more early postoperative complications which was lower than TAH study (28\%). This may be due to good service of the NRH after patients arrive at hospital. The three commonest early postoperative complications other than death were wound infection $23(7.8 \%)$, pneumonia $9(3.0 \%)$ and sepsis $7(2.3 \%)$ which is low when compared with study done in Gondar University Hospital i.e., wound infection (20.6\%), sepsis (17.6\%) and pneumonia (9.9\%) but almost similar study in TAH except sepsis was lower in this study $[7,8]$.

The overall mortality rate of emergency surgically treated acute abdomen was $3.0 \%$ which is lower than study done in Gondar University Hospital (9.3\%), Yirgalem (13.5\%) and Datubo (13.3\%) and TAH (15.3\%) Hospitals. In this study the mean hospital stay of expired patients was 2.2 days and the mean age of the expired patients was 53.6 years. Alost half of the patients who died were operated for bowel obstruction. All of the patients dying with large bowel obstruction had gangrenous sigmoid volvulus $[4,14,15]$.

Two third of all deaths were those patients who came after 2 days

\begin{tabular}{|c|c|c|c|c|c|}
\hline \multirow{2}{*}{ Variables } & \multicolumn{2}{|c|}{ Management outcomes } & \multirow{2}{*}{ COR(95\% Cl) } & \multirow{2}{*}{$\operatorname{AOR}(95 \% \mathrm{Cl})$} & \multirow{2}{*}{$P$ value } \\
\hline & Favorable & Unfavorable & & & \\
\hline$<14$ years & 81 & 8 & $8.94(1.05-76.25)$ & $15.4(1.61-19.98)$ & $.018^{* *}$ \\
\hline \multicolumn{6}{|c|}{ Address } \\
\hline Rural & 147 & 11 & $0.19(.043-0.90)$ & $7.5(1.46-38.46)$ & $.016^{* *}$ \\
\hline \multicolumn{6}{|c|}{ Duration of illness } \\
\hline$>2$ days & 131 & 10 & $0.27(0.07-1.02)$ & $4.33(1.03-18.12)$ & $.045^{\star *}$ \\
\hline \multicolumn{6}{|c|}{ Length of hospital stay } \\
\hline$>$ days & 201 & 5 & $0.39(1.26-12.49)$ & $0.21(0.06-0.73)$ & $.015^{\star *}$ \\
\hline \multicolumn{4}{|l|}{$N^{* *}=s i$} & & \\
\hline
\end{tabular}

Table 4: Multivariate analysis showing independent predictors of management outcomes of non-traumatic acute abdomen from January 2011 -December 2013. 
Citation: Ayenew Z, Gizaw AT, Workneh D, Fentahun N (2016) Outcome of Non-Traumatic Surgical Acute Abdomen in Nekemte Referral Hospital Southwest Ethiopia: A Retrospective Cross-Sectional Study. Surgery Curr Res 7: 282. doi: 10.4172/2161-1076.1000282

Page 5 of 5

of illness and $77.7 \%$ were from rural areas which was similar to TAH study. The mortality rate of $2.1 \%$ for appendicitis $2.6 \%$ of SBO, $4.6 \%$ of LBO and $7.1 \%$ of PPUD was very low when compared with study done in TAH, Gondar University hospital and ZMH $[4,7,8]$.

\section{Conclusion}

In this study we noticed emergency surgical operation for nontraumatic acute abdomen particularly for acute appendicitis was found to be the most common surgical emergency operations performed in NRH. Non traumatic acute abdomen was more common in rural dwellers and male sex. Most of patients experienced post-operative complications were those who came late and related with developing peritonitis.

Acute abdomen is a surgical condition with high rate of morbidity and mortality if not managed timely and appropriately. To alleviate this problem Oromia Regional Health Bureau, particularly NRH should create health awareness on acute abdomen to the general population in general and to all level of health care providers in particular has great importance. As most of the complicated cases with delayed presentation are from rural areas where health institutions with operation theatre and well trained health professionals are not well distributed, cases can be timely handled by properly trained emergency surgeons by assigning them with fully equipped operation theatre. As well since mortality rate was different for different procedures for similar cases, Oromia Regional Health Bureau in collaboration with NRH, should prepare common management protocol for all health workers.

\section{Acknowledgement}

We would like to convey our gratitude to Jimma University College of Health Sciences. We also thank supervisors, data collectors. We appreciate Nekemte Referral Hospital staffs.

\section{References}

1. Faiz O, Banerjee S, Tekkis P, Papagrigoriadiset S, Rennie J, et al. (2007) We still need to operate at night. World Journal of Emergency Surgery 2: 57-65.
2. Gelfand M (1976) The pattern of disease in Africa and the western way of life Trop Doct 6: 173-179.

3. Ali MK (1998) Treatment of sigmoid volvulus: experience in Gondar, north-west Ethiopia. Ethiop Med J 36: 47-52.

4. Zelalem A (2000) Pattern of acute abdomen in Yirgalem Hospital, southern Ethiopia. Ethiop Med J 38: 227-235.

5. Adesunkanmi AR, Agbakwuru EA (1996) Changing pattern of acute intestinal obstruction in a tropical African population. East Afr Med J 73: 727-731.

6. Kotiso B, Abdurahman Z (2007) Pattern of Acute Abdomen in Adult Patients in Tikur Anbessa Teaching Hospital, Addis Ababa, Ethiopia. East Cent Afr J Surg 12: 47-52.

7. Tsegaye S, Osman M, Bekele A (2006) Surgically treated acute abdomen at Gondar university hospital, Ethiopia. East Cent Afr J Surg 12: 53-57.

8. Deneke A, Tadesse B (2003) Incidence, patterns and clinical presentation of acute appendicitis in adults at (ZMH). Ethiop J Health Sci 13: 122 -130.

9. Samuel JC, Qureshi JS, Mulima G, Shores CG, Cairns BA, et al. (2011) An observational study of the etiology, clinical presentation and outcomes associated with peritonitis in Lilongwe, Malawi. World J Emerg Surg 6: 2-5.

10. Demissie M (2001) Small intestine volvulus in Southern Ethiopia. East African Medical Journal 78: 208-211.

11. Sharma AK, Sharma RK, Sharma SK, Sharma A, Soni D (2013) Typhoid intestinal perforation: 24 perforations in one patient. Ann Med Health Sci Res 3: 112-115.

12. Tegegne A (1995) Cultural bowel patterns and sex difference in sigmoid volvulus morbidity in an Ethiopian hospital. Trop Geogr Med 47: 212-215.

13. Ogbonna BC, Obekpa PO, Momoh JT, Ige JT, Ihezue CH (1993) Another look at acute appendicitis in tropical Africa and the value of laparoscopy in diagnosis. Trop Doc 23: 82-99.

14. Warambo MW (1971) Acute volvulus of the small intestine. East Afr Med J 48 209-211.

15. Laal M, Mardanloo A (2009) Acute abdomen; pre and post-laparotomy diagnosis. Int J Collab Res Intern Med Public Health 5: 157-165. 